REGARDS

SUR LECONOMIE ALLEMANDE

BULLETIN ECONOMIQUE DU CRAC

\section{Regards sur l'économie allemande}

Bulletin économique du CIRAC

$101 \mid 2011$

Varia

\title{
Les comptes épargne-temps, instruments de flexibilité
}

\author{
Ines Zapf
}

Traducteur : Solène Hazouard et Isabelle Bourgeois

\section{OpenEdition \\ Journals}

Édition électronique

URL : http://journals.openedition.org/rea/4293

DOI : $10.4000 /$ rea. 4293

ISBN : 978-2-8218-0954-3

ISSN : 1965-0787

Éditeur

CIRAC

Édition imprimée

Date de publication : 10 juin 2011

Pagination : 25-31

ISSN : 1156-8992

\section{Référence électronique}

Ines Zapf, "Les comptes épargne-temps, instruments de flexibilité », Regards sur l'économie allemande [En ligne], 101 | juin 2011, mis en ligne le 01 juin 2013, consulté le 20 avril 2019. URL : http:// journals.openedition.org/rea/4293 ; DOI : 10.4000/rea.4293 


\section{Les comptes épargne- temps, instruments de flexibilité}

Ines Zapf

Contrairement à ce qu'on pouvait redouter, l'impact de la crise financière et économique sur le marché de l'emploi allemand est resté limité. De nombreuses entreprises ont en effet cherché à réduire les effets sur l'emploi du repli, parfois colossal, de leurs commandes et de leur chiffre d'affaires. Cherchant à stabiliser leurs effectifs, elles ont évité le plus possible les mesures de flexibilité externe comme la compression des effectifs, leur préférant toutes les mesures de flexibilité interne - tout particulièrement le chômage partiel ou la liquidation des actifs des comptes épargne-temps (CET). Or si le recours au chômage partiel a joué un rôle important, les comptes épargne-temps ont, quant à eux, joué un rôle majeur dans la stabilisation de l'emploi durant la crise.

Les entreprises allemandes ont été gravement affectées par la crise économique et financière mondiale. Différentes enquêtes révèlent qu'en 2009, près de $50 \%$ d'entre elles estimaient en avoir subi les effets (Bogedan et al., 2009 ; Heckmann et al. estiment cette part à $39 \%$ ). Ceux-ci varient toutefois considérablement d'un secteur à l'autre, les entreprises industrielles ayant été plus lourdement frappées (60\% d'entre elles), du fait de leur orientation à l'export, que les entreprises de services (46,7\%).

Au cours de la crise, nombre d'entreprises ont vu leur volume de commandes et leur CA s'effondrer, la faible demande de biens et de services faisant reculer le PIB allemand de $4,7 \%$ en 2009. C'est dans la production manufacturière que la chute a été la plus nette $(-18,1 \%)$, l'industrie enregistrant dans l'ensemble un recul de 16,7\%. Dans les services, le constat est nettement plus nuancé : alors que le commerce, l'hôtellerie/restauration et les transports ont été affectés par la récession, les services publics et commerciaux ont connu une croissance. Or au regard de la chute du PIB, le marché de l'emploi allemand est resté dans l'ensemble étonnamment robuste. Le nombre d'actifs occupés a même légèrement augmenté globalement en 2009, mais exclusivement en raison de la hausse du temps partiel $(+2,2 \%)$, qui a vu le nombre de salariés passer à 12,4 millions (contre 12,1 millions l'année précédente). Car le nombre d'actifs à temps plein a légèrement baissé $(-1,1 \%)$, passant de 23,7 à 23,5 millions. Ce recul s'explique par une présence plus marquée des temps pleins dans le secteur secondaire, davantage touché par la crise, tandis que l'emploi à temps partiel est plus répandu dans le secteur tertiaire, relativement épargné.

La stabilité de l'emploi et le faible recul du temps plein constatés au niveau macro-économique sont généralement imputés à la stratégie de 'thésaurisation' de la main-d'œuvre et à l'application de différents instruments de flexibilité interne. Ces instruments, parmi lesquels le chômage partiel et la liquidation des actifs de comptes épargne-temps, ont permis aux entreprises de réduire de manière ciblée le temps de travail de leurs collaborateurs et d'accroître ainsi leur flexibilité (Herzog-Stein/Seifert, 2010 ; Schaz/Spitznagel, 2010 ).

Ce constat est confirmé par les statistiques sur le temps de travail réalisées par I'Institut für Arbeitsmarkt- und Berufsforschung (IAB) : le temps de travail annuel des salariés est ainsi passé de 1350,6 à 1309,3 heures entre 2008 et 2009,

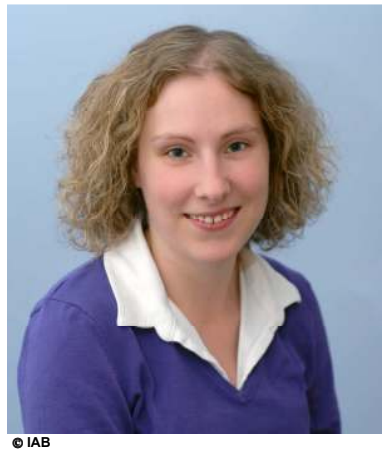

Ines Zapf, sociologue, est chargée de recherche au pôle "Temps de travail et marché de l'emploi " de l'Institut für Arbeitsmarkt-und Berufsforschung (IAB), Nuremberg.

Le nombre d'actifs occupés a légèrement augmenté en 2009

2009 : baisse du nombre d'heures travaillées 
Un outil d'ajustement du temps de travail...

... très prisé dans l'industrie

Des modalités d'application très variées

4 modèles de base soit 41,3 heures de moins par salarié actif $(-3,1 \%)$. Le chômage partiel a eu une part prépondérante dans ce recul (13,4 heures). En effet, alors que le nombre de salariés en chômage partiel n'était que de 101000 en 2008, il a connu une forte hausse et s'établissait à près de 1,1 million en 2009 , ce qui a considérablement accru le volume d'heures chômées. Mais d'autres instruments ont eux aussi contribué à abaisser le temps de travail annuel et ainsi à préserver l'emploi (Fuchs et al., 2010b), ainsi les réductions temporaires de la durée hebdomadaire de travail fixée par convention collective ou usuelle dans l'unité de production (-10,1 heures), la suppression des heures supplémentaires (-7,9 heures) et la liquidation des actifs de comptes épargne-temps ( 7 heures). Durant la récession de 2008/09, l'utilisation des comptes épargne-temps comme instrument de flexibilisation interne en matière d'effectifs a ainsi pour la première fois apporté une contribution notable à la réduction (temporaire) du temps de travail (Herzog-Stein/Seifert, 2010 ), ce que confirment également les constats de Zapf/Brehmer (2010).

\section{Utilité, diffusion et modalités des comptes épargne-temps}

Les comptes épargne-temps avaient été introduits dans les entreprises pour recenser et mieux gérer le temps de travail réellement effectué par les salariés. Lorsque celui-ci excède ou n'atteint pas le niveau convenu, le différentiel est inscrit au compte épargne-temps sous forme d'actifs ou de passifs. Ainsi, pendant les périodes de forte activité, qui se manifestent par un allongement temporaire du temps de travail, sont constitués des actifs de CET qui seront liquidés par la suite lors de phases d'activité réduite, à travers un mécanisme de compensation.

De la sorte, les comptes épargne-temps offrent la possibilité aux entreprises d'organiser de manière flexible le recours à la main-d'œuvre et le temps de travail, en s'adaptant à moindre coût aux variations conjoncturelles et saisonnières. Cela leur permet ainsi d'éviter les périodes creuses et improductives, l'ajustement du temps de travail combinant plus efficacement besoins de maind'œuvre et surcroîts d'activité (Groß/Schwarz, 2010).

Les comptes épargne-temps ont donc connu un véritable essor ces dernières années. Selon le panel d'unités de production de l'IAB, près de $35 \%$ des salariés disposaient d'un CET en 1999. Dix ans plus tard, ils étaient déjà $51 \%$ (Zapf/Brehmer, 2010). Ce dispositif est davantage utilisé dans le secteur de l'industrie et les grandes entreprises, en comparaison avec le secteur des services et les plus petites unités de production (Groß/Schwarz, 2006). En raison de leur large diffusion, de nombreuses entreprises avaient la possibilité d'utiliser les CET comme instrument interne de flexibilité durant la dernière récession. $\mathrm{Ce}$ recours a été favorisé par le fait que lors de la dernière phase de croissance économique, certains CET ont pu atteindre de hauts niveaux d'actifs.

Outre ce facteur, les modalités des CET ont elles aussi joué un rôle essentiel sur leur application pendant la crise. Selon une étude de Groß/Schwarz (2006), les valeurs maximales des actifs de CET sont de près de 69 heures en moyenne, pour des valeurs négatives de -39 heures. La période de compensation des heures positives ou négatives accumulées est de 30 semaines en moyenne. Les possibilités d'organisation des comptes épargne-temps diffèrent toutefois considérablement d'une entreprise à l'autre, et il en existe aujourd'hui de nombreuses variantes.

Seifert (2001) identifie quatre modèles de base: les comptes d'horaires variables (Gleitzeitkonten), les comptes d'heures supplémentaires (Überstundenkonten), les modèles d'accumulation (Ansparmodelle) et les modèles d'échelle (Bandbreitenmodelle). Les comptes d'horaires variables sont considérés comme l'archétype des comptes épargne-temps. Ils avaient été introduits dès les années 1960, alors qu'on cherchait à abolir l'obligation de ponctualité sur le 
lieu de travail, à accroître l'autonomie du temps de travail et à individualiser le temps de travail. Dans les comptes d'horaires variables, le début et la fin de la durée de présence quotidienne au travail sont libres, mais celle-ci doit respecter les horaires de base communs. La constitution d'actifs ou de passifs est limitée, la compensation s'effectuant presque en temps réel, ce qui aura limité le recours aux comptes d'horaires variables durant la récente récession. Les comptes d'heures supplémentaires permettant seulement la constitution d'actifs, leur mise en œuvre comme instrument de gestion de crise est selon toute vraisemblance elle aussi restée limitée dans le temps. Les modèles d'accumulation ne permettent eux aussi que la constitution d'actifs ; et ce dans le but de suivre une qualification ou une formation continue durant l'activité, de prendre un congé parental ou de préparer une retraite anticipée. Certes, l'ampleur des actifs accumulés permet en théorie de prolonger d'autant la période d'activité, mais la légitimation particulière de ces comptes s'y oppose dans la réalité.

Enfin, les modèles d'échelle offrent une flexibilité supérieure étant donné que les plafonds d'actifs et de passifs sont particulièrement élevés. Quant à la période de compensation, qui dure souvent une année, elle les rend particulièrement aptes à pallier les phases de faiblesse non seulement saisonnière, mais aussi conjoncturelle (Hildebrandt 2007 ; Seifert 2001 ).

\section{Modèles d'échelle : les mieux adaptés} en période de récession

Source des données : une enquête de l'institut WSI menée à l'été 2009

Dans le contexte de la crise économique et financière, l'Institut de recherches économiques et sociales (Wirtschaftsund Sozialwissenschaftliches Institut, WSI) de la Fondation Hans-Böckler, émanation de la Confédération syndicale DGB, a mené du 14 juillet au 28 septembre 2009 une enquête auprès des conseils d'établissement (Betriebsrat) dans le secteur privé. Outre des questions générales sur la situation actuelle de l'entreprise et son évolution passée et à venir, ce sondage comprenait des questions portant plus précisément sur les mesures de préservation de l'emploi pendant la crise, comme le chômage partiel et la liquidation des actifs de comptes épargne-temps. L'enquête s'intéressait par ailleurs aux accords visant à préserver l'emploi et à leur efficacité pendant la crise, à la qualification, à l'évaluation des mesures de préservation de l'emploi et aux perspectives qu'elles offrent ainsi qu'à des données générales concernant l'entreprise.

Les entreprises du panel étaient principalement issues des branches et secteurs suivants : matières premières/biens de production, biens d'investissement et de consommation durable, biens de consommation, BTP, commerce (et réparations), transports et télécommunications, crédit et assurances, autres services publics et commerciaux. Au total, les chercheurs de l'institut WSI disposaient de données issues de 2324 entreprises de 20 salariés et plus, disposant d'un conseil d'établissement; l'enquête est représentative des différents secteurs et catégories d'entreprises (infas 2009). Les entreprises participantes occupaient au total près de 743000 salariés, soit $2,1 \%$ de l'ensemble des salariés allemands tous secteurs confondus.

Pour résumer, les comptes épargne-temps peuvent être mis en œuvre en cas de baisse saisonnière ou conjoncturelle de la demande, afin d'éviter (ou du moins de retarder) les suppressions d'emplois. La décision de procéder à des licenciements n'est en effet pas sans conséquences pour une entreprise : elle peut être préjudiciable en termes d'image, et engendrer des coûts non négligeables. Ces coûts peuvent augmenter encore en cas d'embauche ou de réembauche, en raison des frais supplémentaires de recherche, de sélection de candidats et/ou de frais de formation (Bellmann/Gewiese, 2004 . A l'inverse, en cas d'accroissement de la demande et de forte activité, ils permettent d'accumuler les heures supplémentaires travaillées sous forme d'actifs de CET et de répartir le surcroît de travail entre les salariés. Mais cette mesure réduit ainsi les possibilités d'embauche. Après les effets stabilisateurs qu'a eue la flexibilité interne offerte par les comptes épargne-temps pendant la récession pourrait ainsi suivre une phase de croissance sans emploi (« jobless growth ») dans les entreprises concernées.

\section{Les comptes épargne-temps, instruments de flexibilité durant la crise}

Pour éviter ou au moins retarder le recours aux mesures de flexibilité externe comme la suppression de postes, les entreprises disposent donc de plusieurs

Un vaste éventail de mesures de flexibilité interne 
Prédilection pour la liquidation des comptes épargne-temps

Ouverture de comptes épargne-temps en réponse à la crise possibilités pour accroître leur flexibilité interne en matière d'effectifs ; et elles utilisent généralement plusieurs mesures à la fois.

Les mesures de flexibilité interne s'offrant aux entreprises sont notamment: le chômage partiel, la liquidation des actifs ou pour la constitution de passifs de comptes épargne-temps, les dispositions relatives aux congés ou la réduction des prestations sociales prévues par l'entreprise. S'y ajoutent des mesures personnelles et organisationnelles, comme les reconversions et les mutations, les baisses de salaire ou les modifications du temps de travail, par exemple une réduction du temps de travail hors du cadre du chômage partiel.

Dans cet éventail, les entreprises interrogées ont opté en premier lieu (33,9\%) pour la liquidation des actifs ou à la constitution de passifs de CET sur la période allant du $3^{\mathrm{e}}$ trimestre 2008 au $3^{\mathrm{e}}$ trimestre 2009. De nombreuses entreprises ont également adopté des mesures personnelles et organisationnelles $(29,1 \%)$, ou ont recouru au chômage partiel (23,8 \%). Peu d'entreprises en comparaison ont déclaré avoir modifié le temps de travail $(10,8 \%)$ ou réduit les prestations sociales (7,9\%; plusieurs réponses possibles).

Entre le $3^{\mathrm{e}}$ trimestre 2008 et le $3^{\mathrm{e}}$ trimestre 2009, la plupart des entreprises interrogées ont instauré des comptes épargne-temps en vue de préserver l'emploi. Cela peut avoir été motivé par la crise. Néanmoins, indépendamment de la crise, les accords d'entreprise peuvent prévoir une liquidation des actifs de comptes épargne-temps dans l'objectif du maintien de l'emploi : en contrepartie des concessions accordées par les salariés, les emplois sont sécurisés et les transferts, délocalisations ou licenciements évités (Zapf/Herzog-Stein, 2011).

Une nette majorité des entreprises ayant recouru à la liquidation des actifs ou à la constitution de passifs de CET affirme toutefois que ces mesures ont été prises en réaction à la crise : c'est la motivation avancée par près des trois quarts de celles qui ont liquidé les heures épargnées; un quart seulement avance d'autres raisons. Parmi les entreprises se disant affectées par la crise, $86,4 \%$ ont puisé dans les actifs de CET pour cette raison, 13,6\% seulement pour d'autres raisons. Mais du fait des relations d'interdépendance au sein de l'économie, les entreprises qui n'étaient pas directement affectées par la crise ont elles aussi procédé à la liquidation d'actifs de CET en raison de la crise, à plus faible échelle toutefois (43,2\%). Pour $56,8 \%$ de ces dernières, la réduction des heures suivait d'autres motifs.

Actifs de CET moyens des salariés avant et après liquidation ( $2^{e}$ trim. 2008 et $3^{e}$ trim. 2009, part des entreprises en \%)

\begin{tabular}{|lcc|}
\hline & $\begin{array}{c}\text { Actifs de CET moyens avant liquidation } \\
\left(2^{e} \text { trimestre 2008) }\right.\end{array}$ & $\begin{array}{c}\text { Actifs de CET moyens après liquidation } \\
\left(3^{e} \text { trimestre 2009) }\right.\end{array}$ \\
Absence d'actifs & 0,0 & 26,2 \\
de $\mathbf{1}$ à $\mathbf{1 9}$ heures & 8,0 & 22,7 \\
de $\mathbf{2 0}$ à $\mathbf{3 9}$ heures & 19,3 & 21,4 \\
de $\mathbf{4 0}$ à $\mathbf{5 9}$ heures & 22,2 & 17,1 \\
$\mathbf{6 0}$ heures et plus & 50,5 & 12,6 \\
Total & 100,0 & 100,0 \\
\hline
\end{tabular}

Source : calculs de l'IAB sur la base de l'enquête WSI menée en 2009 auprès de conseils d'établissement (résultats pondérés). Base de calcul : uniquement les entreprises concernées par la crise économique depuis juillet 2008, qui pour cette raison ont liquidé depuis des actifs de CET dont le solde est actuellement nul voire positif. Seules sont considérées les entreprises donnant des informations sur le bilan et la liquidation moyenne des actifs au moment de l'enquête, $\mathrm{N}=361$.

Forte réduction des actifs moyens de CET dans les entreprises touchées par la crise
Dans les entreprises qui se disaient affectées par la crise depuis juillet 2008 et qui avaient pour cette raison liquidé des actifs de CET, cette pratique a entraîné un net recul des actifs moyens accumulés par les salariés. Dans certaines entreprises, ceux-ci ont même été épuisés dans leur intégralité. En revanche avant le début de la crise, les comptes épargne-temps des salariés affichaient en moyenne un solde positif dans l'ensemble des entreprises ; et du fait du taux élevé d'utilisation des capacités durant la période de forte croissance, certains volumes d'actifs de CET avaient atteint des niveaux considérables: dans la moitié des entreprises, il se situait à au moins 60 heures. La liquidation des 
actifs s'est soldée, dans une entreprise sur quatre, par l'utilisation de la totalité des heures cumulées; dans une sur huit seulement, le volume moyen des actifs restait malgré tout considérable ( 60 heures et plus).

La situation était à peu près la même dans tous les branches d'activité. Toutefois avant liquidation, le secteur secondaire rassemblait plus d'entreprises avec un actif moyen de 60 heures et plus $(53,3 \%)$ que le secteur tertiaire $(46,7 \%)$. Mais après liquidation pendant la crise, les entreprises industrielles ont davantage épuisé leurs comptes d'actifs $(29,5 \%)$ que celles des services $(21,5 \%)$.

Moyenne des actifs de CET liquidés par salarié selon les secteurs (du $3^{e}$ trim. 2008 au $3^{e}$ trim. 2009, part des entreprises en \%)

\begin{tabular}{|lccc|}
\hline & Secteur secondaire & Secteur tertiaire & Total \\
jusqu'à $\mathbf{1 9}$ heures & 17,1 & 25,2 & 20,5 \\
de $\mathbf{2 0}$ à $\mathbf{9 9}$ heures & 27,1 & 38,4 & 31,9 \\
de $\mathbf{4 0}$ à $\mathbf{5 9}$ heures & 23,8 & 19,2 & 21,9 \\
$\mathbf{6 0}$ heures et plus & 31,9 & 17,2 & 25,8 \\
Total & 100,0 & 100,0 & 100,0 \\
\hline
\end{tabular}

Source : calculs de I'IAB sur la base de l'enquête WSI menée en 2009 auprès de conseils d'établissement (résultats pondérés). Base de calcul : uniquement les entreprises concernées par la crise économique depuis juillet 2008, qui pour ponderes). Base de calcul : uniquement les entreprises concernees par la crise économique depuis juillet 2008, qui pour les entreprises donnant des informations sur le bilan et la liquidation moyenne des actifs au moment de l'enquête, $\mathrm{N}=361$.

La crise a mené à une forte liquidation d'actifs dans les entreprises affectées : en moyenne, les actifs d'un salarié y sont passés de près de 72 à 27 heures entre juillet 2008 et le troisième trimestre 2009 , soit une baisse de quelque 45 heures $(-62,5 \%)$. La plupart des entreprises ont liquidé entre 20 et 39 heures. Le secteur secondaire étant fortement orienté vers les exportations, de nombreuses entreprises y ont été affectées par la crise, et elles y ont beaucoup plus largement liquidé les actifs de CET que dans le tertiaire. Les entreprises industrielles ont ainsi supprimé en moyenne 50 heures d'actifs par salarié, cette suppression allant jusqu'à 60 heures et au-delà pour la majorité d'entre elles. La moyenne s'élevait à près de 38 heures pour les entreprises des services qui, pour la plupart, ont liquidé entre 20 et 39 heures d'actifs de CET.

Intensité de la liquidation d'actifs de CET dans les entreprises par secteur (du $3^{\mathrm{e}}$ trim. 2008 au $3^{\mathrm{e}}$ trim. 2009, part des entreprises en \%)

\begin{tabular}{|lccr|}
\hline Personnel concerné & Secteur secondaire & Secteur tertiaire & Total \\
\hline jusqu'à 20 \% & 7,7 & 10,7 & 8,9 \\
de 21 \% à 60\% & 29,2 & 32,0 & 30,4 \\
plus de 60 \% & 63,2 & 57,3 & 60,7 \\
Total & 100,0 & 100,0 & 100,0 \\
\hline
\end{tabular}

Source : calculs de I'IAB sur la base de l'enquête WSI menée en 2009 auprès de conseils d'établissement (résultats pondérés). Base de calcul : uniquement les entreprises concernées par la crise économique depuis juillet 2008, qui pour cette raison ont liquidé depuis des actifs de CET dont le solde est actuellement nul voire positif. Seules sont considérées les entreprises donnant des informations sur le bilan et la liquidation moyenne des actifs au moment de l'enquête, $\mathrm{N}=361$.

Outre une forte diminution des actifs moyens, les entreprises ont atteint un haut niveau de flexibilité en raison de la large diffusion des CET auprès de leur personnel. Car la liquidation des actifs ne s'est pas limitée à quelques groupes de salariés isolés, mais s'est appliquée à une nette majorité du personnel dans nombre d'entreprises. Ainsi dans six entreprises sur dix, plus de $60 \%$ des salariés ont vu leurs actifs se réduire. A l'inverse, rares sont les entreprises où ce phénomène est demeuré circonscrit. Eu égard à l'intensité de la liquidation d'actifs, les différences entre secteurs sont minimes : une majorité de salariés a été concernée, aussi bien dans l'industrie que dans les services. Cependant la proportion des entreprises dans lesquelles la majeure partie des salariés a connu une réduction des actifs de CET était plus élevée dans le secteur secondaire, la différence avec le secteur tertiaire restant minime.

Dans nombre d'entreprises, la réglementation en matière de comptes épargnetemps est organisée de telle sorte que la mise en place de passifs peut venir s'ajouter à celle des actifs de CET. La limite maximale est en règle générale
Une moyenne de $\mathbf{5 0}$ heures liquidées dans l'industrie

La liquidation d'actifs a concerné la majorité du personnel

Les entreprises affectées par la crise ont moins recouru à l'accumulation de passifs 
nettement plus élevée pour les heures accumulées que pour les heures dues. Lorsque les entreprises concernées par la crise offraient par principe la possibilité de cumuler des valeurs négatives de CET, celle-ci s'accompagnait généralement (dans $86,9 \%$ des cas) d'une limite maximale. Dans plus de la moitié des entreprises, cette limite était de 60 heures ou plus. Dans quelques rares entreprises, elle était de moins de 20 heures. Ce constat laisse supposer que, dans le contexte de la crise, les entreprises ont étendu les limites maximales de passifs de CET afin d'accroître encore au besoin leur flexibilité interne. En moyenne, jusqu'à 79 heures dues pouvaient ainsi être accumulées sur les comptes épargne-temps.

Malgré l'existence de telles dispositions, très peu seulement d'entreprises affectées par la crise ont recouru à l'accumulation de passifs $(4,5 \%)$, lui préférant de loin la liquidation des actifs de CET. II y a plusieurs explications à cela : les actifs doivent être totalement épuisés avant que puissent s'accumuler les heures dues, ce qui était le cas pour seulement un bon quart des entreprises au moment de l'enquête. D'un autre côté, dans de nombreuses entreprises, l'évolution de la réglementation en matière de chômage partiel (voir REA 90/09) a contribué à plaider en faveur de cet instrument plutôt que pour la mise en place de passifs de CET. Car si depuis février 2009, les actifs de CET doivent toujours être épuisés pour pouvoir accéder aux allocations de chômage partiel, il n'est plus nécessaire d'accumuler les heures dues.

Néanmoins, les quelques entreprises ayant mis en place ce système de passif de CET y ont eu massivement recours : en moyenne, chaque salarié a totalisé près de 46 heures négatives, et plus de la moitié des salariés était concernée.

DURANT LA CRISE, NOMBRE D'ENTREPRISES ONT ACCRU LEUR FLEXIBILITÉ à l'aide de diverses mesures. Les instruments de flexibilité interne en matière d'effectifs ont été particulièrement plébiscités afin de garantir l'emploi en essayant de diminuer le recours aux mesures de flexibilité externe. En ce qui concerne les mesures de flexibilité interne, les entreprises interrogées ont principalement eu recours à la liquidation des actifs ou à la constitution de passifs de CET. Elles ont par ailleurs opté aussi pour le chômage partiel ou les mesures personnelles et organisationnelles.

Si de nombreuses entreprises ayant instauré des comptes épargne-temps ont procédé à la liquidation de leurs actifs, peu d'entre elles ont à l'inverse choisi d'accumuler les heures dues. Cela peut s'expliquer par les risques inhérents en termes de coûts ainsi que par la volonté de ne pas mettre davantage à contribution les salariés. Car les heures négatives accumulées représentent une prestation financière exécutée par l'entreprise avant la contre-prestation de ses salariés. Quant aux salariés, ils doivent s'acquitter par la suite des heures dues en effectuant des heures supplémentaires et équilibrer ainsi le CET dans un délai donné, établi au niveau de l'entreprise.

Le haut degré de flexibilité montré par les entreprises allemandes lors de la récente récession s'explique en majeure partie par l'accumulation d'actifs sur les CET pendant les années de croissance qui l'ont précédée. La liquidation des actifs de comptes épargne-temps et partant, le recul global du temps de travail ont contribué à stabiliser l'emploi. Les comptes épargne-temps peuvent dès lors être considérés comme un instrument efficace pour surmonter les crises économiques. Il permet sinon d'éviter, du moins de réduire considérablement les licenciements, et de garder au sein de l'entreprise le capital humain patiemment accumulé, ce qui permet en retour d'épargner d'éventuels coûts de réembauche. Dans le même temps, la sécurité de l'emploi s'accroît pour les salariés. Mais les chômeurs voient aussi se réduire leurs perspectives de réintégrer le marché de l'emploi lors d'une reprise de la conjoncture. Les " insiders » de l'entreprise sont ainsi largement protégés, souvent au détriment des " outsiders ».

La flexibilité via les comptes épargne-temps permet aussi aux entreprises d'accroître rapidement leur taux de capacité et leur productivité. C'est ainsi que, 
contre toute attente, l'économie allemande est sortie de la récession dès 2010 , avec une croissance de $3,6 \%$ du PIB en 2010 , ce qui aura des effets multiplicateurs bénéfiques aux chômeurs, puisqu'une forte croissance est génératrice d'emplois. L'an passé, le nombre d'actifs occupés a de nouveau légèrement augmenté.

Rappelons toutefois pour conclure que les résultats présentés reposent sur les réponses des conseils d'établissement dans les entreprises de 20 salariés et plus ; les constats de cette étude ne sont dès lors représentatifs que pour les entreprises de cette taille et disposant d'un conseil d'établissement. Pour les entreprises interrogées, la liquidation d'actifs ou la constitution de passifs de CET représentent les mesures les plus utilisées pour préserver l'emploi lors de la crise. Du fait de la fonction régulatrice des conseils d'établissement et de l'intérêt qu'ils témoignent pour la préservation des emplois permanents, il n'est pas à exclure que ces représentations élues du personnel ont encouragé le recours à cet instrument. Quant aux entreprises dépourvues de conseil d'établissement, aucune donnée n'est à ce jour disponible. On peut toutefois supposer qu'elles aussi ont eu recours aux comptes épargne-temps pour préserver l'emploi.

(Traduction I. Bourgeois et S. Hazouard)

\section{Indications bibliographiques}

-Bellmann L., Gewiese T., «Entwicklung der Arbeitszeitkonten in Deutschland », in: Arbeit und Beruf, $\mathrm{n}^{\circ} 11 / 2004,2004$, pp. 329-331

- Bogedan C., Brehmer W., Herzog-Stein A., Betriebliche Beschäftigungssicherung in der Krise. Eine Kurzauswertung der WSI-Betriebsrätebefragung 2009, Düsseldorf, 2009

- EICHHORSt W., MARX P., "Le chômage partiel, amortisseur social de la crise? », Regards sur l'économie allemande, $n^{\circ}$ 90/2009

- Fuchs J., Hummel M., Klinger S., Spitznagel E., Wanger S., ZiKa G., « Prognose 2010/2011: Der Arbeitsmarkt schließt an den vorherigen Aufschwung an », IAB-Kurzbericht, n 18/2010, Nuremberg, 2010b

- Groß H., Schwarz M., Betriebs- und Arbeitszeiten 2005. Ergebnisse einer repräsentativen Betriebsbefragung, Dortmund, 2006

- GROß H., SchwARz M., Arbeitszeit, Altersstrukturen und Corporate Social Responsibility: Eine repräsentative Betriebsbefragung, VS-Verlag für Sozialwissenschaften, Wiesbaden, 2010

- Heckmann M., Kettner A., Pausch S., Szameitat J., Vogler-Ludwig K., « Unternehmensbefragung im II. Quartal 2009: Wie Betriebe in der Krise Beschäftigung stützen », IAB-Kurzbericht, $n^{\circ}$ 18/2009, Nuremberg, 2009

- Herzog-Stein A., Seifert H., « Der Arbeitsmarkt in der Großen Rezession - Bewährte Strategien in neuen Formen », WSI-Mitteilungen, ${ }^{\circ} 11,2010$

- Hildebrandt E., "Langzeitkonten, Lebensplanung und Zeithandeln », in Hildebrandt E. (Hg.), Lebenslaufpolitik im Betrieb. Optionen zur Gestaltung der Lebensarbeitszeit durch Langzeitkonten, Berlin, 2007

- INStitut FÜR ANGEWANDTE SOZIALWISSENSChaft GmbH (INFAS), WSI Betriebsrätebefragung 2009. Sondererhebung „Beschäftigungssicherung“. Methodenbericht, Bonn, 2009

- Schaz P., Spitznagel E., « Makroökonomische Dynamik von Arbeitsmärkten. Ein Vergleich interner und externer Flexibilitäten in den USA und in Deutschland », WSI-Mitteilungen, $n^{\circ}$ 12, 2010

-SEIFERT H., „Zeitkonten: Von der Normalarbeitszeit zu kontrollierter Flexibilität“, WSI-Mitteilungen, $\mathrm{n}^{\circ} 2,2001, \mathrm{pp} .84-91$

-ZAPF I., BREHMER W., «Flexibilität in der Wirtschaftskrise : Arbeitszeitkonten haben sich bewährt », IAB-Kurzbericht, $n^{\circ}$ 22/2010, Nuremberg, 2010

-ZAPF I., HeRzog-Stein A., « Betriebliche Einsatzmuster von Arbeitszeitkonten während der Großen Rezession », in WSI-Mitteilungen, $\mathrm{n}^{\circ}$ 2, 2011. 